\title{
Los cambios en el consumo de alcohol de una persona influyen sobre el comportamiento de sus familiares y amigos
}

\author{
Alcohol consumption changes in a person influence the behaviour of his relatives and friends
}

Rosenquist J y col. Ann Intern Med 2010;152:426-433.

\section{Objetivo}

Explorar cuantitativamente si el comportamiento de consumo de alcohol se extiende de una persona a otra en una gran red social de compañeros de trabajo, hermanos, esposos y vecinos.

\section{Diseño, lugar y participantes}

Cohorte longitudinal de redes* sociales con 32 años de seguimiento anidada en la de Factores de Riesgo Cardiovasculares de Framingham, Masachussets, EE.UU. Del total de 12.067 participantes evaluados siete veces entre 1971 y 2003,5124 eran participantes principales y el resto sus lazos sociales.

\section{Mediciones}

Auto-reporte del consumo de alcohol y de lazos en la red social, medidos en cada corte temporal. Los lazos sociales fueron auto-informados por los participantes del estudio (principales) y considerados según grados de separación. Así, si un participante principal (A) informaba que B era su amigo, y su amigo $B$ informaba que $C$ era su amigo, $C$ se convertía en un lazo social de segundo grado para A. Se consideró consumo excesivo a mas de una bebida estándar diaria para mujeres y dos para varones.

\section{Resultados principales}

Fue constatada la presencia de agrupamientos de abstemios y bebedores durante todas las evaluaciones realizadas, extendiéndose los mismos hasta tres grados de separación. Estos agrupamientos no fueron sólo el resultado de la formación selectiva de lazos sociales entre bebedores, sino que también parecieron reflejar influencias interpersonales. Por ejem- plo y luego de controlar por los potenciales confundidores, los cambios en el comportamiento de consumo de alcohol de un miembro de la red social de un participante principal se asociaron a un aumento en la probabilidad de que el participante principal cambiara su comportamiento de consumo, con una influencia levemente mayor para el consumo excesivo (50\%; IC95\% 40 a 62 para un lazo de primer grado) que para la abstención (29\%; IC95\% 23 a 36).

Además, se halló una disminución de la influencia a medida que los lazos sociales se hacían más distantes, manteniéndose una significancia estadística hasta tres grados de separación y contrastando este efecto, con la falta de disminución respecto de la distancia geográfica, dado que un amigo que vivía en el mismo barrio tenía el mismo efecto que otro que se encontraba a miles de kilómetros de distancia. Este efecto de influencia en el comportamiento se observó para familiares y amigos con hasta tres grados de separación de la red social, pero no para vecinos y compañeros de trabajo.

\section{Conclusiones}

Cualquier modificación en el consumo de familiares y amigos de una persona influenciaría el propio. Estos resultados tienen implicancias para las intervenciones clínicas y de salud pública, brindando también soporte empírico a las intervenciones a nivel grupal destinadas a disminuir la ingesta problemática.

Palabras claves: consumo de alcohol, alcohol y red social, entorno. Key words: alcohol consumption, alcohol, social network, context.

Fuente de financiamiento: Instituto Nacional de Salud de los Estados Unidos de Norteamérica y Fundación Robert Wood Johnson. El estudio Framingham del corazón es avalado por el Instituto Nacional Norteamericano de Corazón Pulmón y Sangre.

\section{Comentario}

Entre los profesionales de la salud existe la creencia arraigada que se expresa en el dicho: “....si ves un alcohólico en una familia, busca más que lo encontrarás....". Por otro lado, el alcohol como fenómeno social es ampliamente conocido en nuestra cultura, mientras que la influencia social sobre los comportamientos de consumo de alcohol siempre fue objeto de análisis, teoría y estudio.

En este sentido y con sus 40 años de historia, el estudio del corazón de Framingham nunca deja de sorprendernos por la variedad y calidad de los datos que nos ofrece, aportando evidencia novedosa que sugiere algún tipo de causalidad, más allá de un simple agrupamiento selectivo entre bebedores. Podríamos suponer además que para las culturas de origen latino en las que los lazos sociales cobran mayor importancia, estas influencias podrían ser mayores.

Respecto de su metodología, llama la atención que hayan definido los niveles de consumo de alcohol de manera diferente a la que propone la Organización Mundial de la Salud ${ }^{1}$ para investigaciones de este tipo (considerar consumo excesivo a cinco o más bebidas estándar para varones y a cuatro para mujeres) y el bajo porcentaje de abstemios en la población estudiada.

\section{Conclusiones de los comentadores}

Este potencial efecto multiplicador de las intervenciones contra los problemas relacionados con el alcohol, nos hace pensar en un efecto similar al de la vacuna Sabin, que no solo beneficia a quien recibe directamente la intervención, sino también a aquellos individuos que están en contacto íntimo con el vacunado. Esperemos que estos resultados se vean reflejados en nuevos estudios que nos permitan mejorar las intervenciones en las poblaciones en riesgo por el consumo de alcohol.

Esteban Lucas Figueroa [ Servicio de Medicina Familiar del Hospital Italiano de Buenos Aires, Universidad Católica Santa María de los Buenos Aires y Grupo Atención Primaria SRL grupoatencionprimaria@gmail.com]

Mariana Cremonte [ Universidad de Mar del Plata y Investigadora adjunta del CONICET mcremont@mdp.edu.ar]Universidad Nacional de Mar del Plata.]

Figueroa E y Cremonte M. Los cambios en el consumo de alcohol de una persona influyen sobre el comportamiento de sus familiares y amigos. Evid Act Pract Ambul 14(2). 53. Abr-Jun 2011. Comentado de: Rosenquist $\mathbf{J}$ y col. The Spread of Alcohol Consumption Behavior in a Large Social Network. Ann Intern Med 2010;152:426-433. PMID: 20368648.

\section{Referencias}

1. Wordl Health Organization. Global Status Report on Alcohol and Health 2011.

Disponible en URL: http://www.who.int/substance_abuse/publications/global_alcohol_report/en/index.html (último acceso 04/04/11). 